\title{
BMJ Open Is there a correlation between children's outdoor active mobility behaviour and neighbourhood safety? A systematic review of the evidence
}

\author{
Roula Zougheibe (D , ${ }^{1}$ Beverly Jepson, ${ }^{2}$ Richard Norman, ${ }^{3}$ Ori Gudes, ${ }^{4}$ \\ Ashraf Dewan ${ }^{1}$
}

To cite: Zougheibe R, Jepson B, Norman $\mathrm{R}$, et al. Is there a correlation between children's outdoor active mobility behaviour and neighbourhood safety? A systematic review of the evidence. BMJ Open 2021;11:e047062. doi:10.1136/ bmjopen-2020-047062

- Prepublication history and additional supplemental material for this paper are available online. To view these files, please visit the journal online (http://dx.doi.org/10.1136/ bmjopen-2020-047062).

Received 23 November 2020 Accepted 24 June 2021

Check for updates

(c) Author(s) (or their employer(s)) 2021. Re-use permitted under CC BY-NC. No commercial re-use. See rights and permissions. Published by BMJ.

${ }^{1}$ School of Earth and Planetary Sciences, Curtin University,

Perth, Western Australia, Australia

${ }^{2}$ School of Allied Health, Curtin University, Perth, Western Australia, Australia

${ }^{3}$ School of Public Health, Curtin University, Perth, Western Australia, Australia

${ }^{4}$ School of Population Health, UNSW, Sydney, New South Wales, Australia

Correspondence to Roula Zougheibe; roula.zougheibe@postgrad. curtin.edu.au

\section{ABSTRACT}

Objective To identify, summarise and evaluate evidence on the correlation between perceived and actual neighbourhood safety (personal and road danger) and diverse forms of outdoor active mobility behaviour (ie, active play, exercise, and travel) among primary-schoolaged children.

Design A systematic review of evidence from observational studies exploring children's active mobility behaviour and safety.

Data sources Six electronic databases were searched: Google Scholar, PubMed, Scopus, Science Direct, ProQuest and Web of Science from study inception until July 2020.

Data extraction and synthesis Study selection and quality assessment were conducted independently by two reviewers. We expanded on a quality assessment tool and adopted a vote-counting technique to determine strength of evidence. The outcomes were categorised by individual, family and neighbourhood levels.

Results A total of 29 studies were included, with a majority of cross-sectional design. Higher parental perceived personal safety correlated with increased children's active mobility behaviour, but most commonly in active travel (eg, independent walking or cycling to a local destination). Increased concerns regarding road danger correlated with a decrease in each type of children's active behaviour; active travel, play and exercise. However, these correlations were influenced by child's sex/gender, age, car ownership, neighbourhood types, across time, and proximity to destination. Limited or inconclusive evidence was found on correlate of children's outdoor active mobility behaviour to 'stranger danger', children's perceived personal safety, race/ethnicity, socioeconomic status or measured safety.

Conclusion Children are restricted by perception of safety. Encouraging children's active travel may require future strategies to address characteristics relevant to types of the neighbourhood that promote a high sense of personal safety. Children and parents may embrace other types of active mobility behaviour if road danger is mitigated. Sex/gender and age-specific interventions and redesign of public places could lead to childfriendly cities. Future studies may benefit from adopting validated measurement methods and fill existing research gaps.
Strengths and limitations of this study

- This systematic review adapted a methodological quality appraisal that integrated studies' reporting and measurement methods along with a votecounting technique to understand the strength of the evidence.

- To our knowledge, this is the first systematic review that comprehensively synthesises evidence on neighbourhood safety correlates to forms of primary school-aged children's outdoor active mobility behaviours.

- Peer-reviewed studies published in languages other than English were excluded, and meta-analysis review was not possible due to the heterogeneity in studies measurement methods and outputs.

\section{INTRODUCTION}

Children's daily active mobility behaviour is linked with profound long-term positive outcomes. ${ }^{1}$ Recommended daily level of children's physical activity is primarily achieved through various forms of structured (ie, organised sport) or unstructured active behaviour (ie, active play in open spaces, walking, cycling or scootering to school and other local destinations). ${ }^{23}$ Neighbourhood, the place where a child's majority of daily routines occur, has been identified as a primary venue for children to meet the daily physical activity recommendations. ${ }^{2}$ However, variability in the neighbourhood (ie, residential, commercial, industrial and agricultural) have been found to affect types and extent of children's various shapes of active behaviour. ${ }^{45}$ For example, different types of neighbourhood were linked to changed levels of active transportation in children aged 10-11 years living in Finnish urban areas. ${ }^{6}$ Canadian children travel longer distance actively in residential or commercial areas than other types of neighbourhood. ${ }^{5}$ Urban 
and road structures in The Netherlands reversed the negative effect of reduced active travel with longer distances. ${ }^{7}$ Nevertheless, neighbourhood impact on other aspects of children's behaviour such as play and peer interaction is mixed. ${ }^{8}$

Safety, including personal and road danger, ${ }^{9}{ }^{10}$ is a complex concept. ${ }^{3}$ Perceived (by parents or children) or measured safety (actual crime and traffic danger) may create obstacles resulting in children's lower confidence in exploring their surroundings, ${ }^{2}$ or contributing to their community. ${ }^{11}$ Lack of safety may have contributed to urban streets and public spaces becoming increasingly inhospitable environments. With more people living in urban settings than ever before, ${ }^{12}$ and increasing safety concerns, children's territory for active outdoor mobility behaviour, such as walking, playing, cycling and scootering, has shrunk. ${ }^{13-16}$ The damage caused by restricting children's active mobility behaviour may extend beyond their immediate physical health, ${ }^{17}$ to overall social skills ${ }^{18}$ and healthy brain development. ${ }^{19}$

Nevertheless, reviews that addressed neighbourhood safety and children's active behaviour have reported conflicting results. The disagreements could be due to: narratively investigating safety, ${ }^{3}$ examining one shape of active behaviour such as active school travel ${ }^{90-23}$ or independent mobility ${ }^{24}$ or combinedly addresses safety with other environmental correlates and from a wide age range of children age (three or five to 18 years). ${ }^{16} 2526$ Over and above that, variability in measurement methods and output adds complexity in evaluating available evidence. $^{2022}$ The absence of suitable checklists that account for measurement methods when assessing the quality of observational studies, ${ }^{24}$ called research for alternatives approach to increase confidence in the synthesis of studies outcome ${ }^{2022}$ and inform evidence-based policy decisions. On the other hand, active school travel is a significant contributor to overall child active behaviour, ${ }^{27}$ but other forms of activity incorporated into children's daily routines ${ }^{24}$ are vital contributors to children's daily movement targets. ${ }^{28}$ No review up to now has exclusively focused on the varying impact of measured and perceived safety in urban settings across primary-school-aged children's various forms of daily active mobility behaviours. Thus, this review aims to (1) systematically synthesise evidence of correlations between primary-school-aged children's outdoor active mobility behaviour (COAMB) and neighbourhood measured and perceived safety, and (2) derive the strength of the evidence by evaluating quality and methodological measurement method for each study and vote counting technique as justified from previous reviews.

\section{METHOD}

This systematic review was conducted according to the Preferred Reporting Items for Systematic Reviews and Meta-Analysis (PRISMA). The corresponding PRISMA checklist was completed during the review, as illustrated in online supplemental file 1 . Though the search was planned, no protocol was registered.

\section{Search strategy}

A keyword search was designed and conducted to identify all relevant studies in six electronic databases: Google Scholar, PubMed, Scopus, Science Direct, ProQuest and Web of Science. All databases were searched between March 2019 and July 2020. The search algorithm included all possible combinations of keywords. Terms pertaining to this review were grouped by (1) target population, (2) AND active behaviour, AND (3) neighbourhood safety, OR (4) moderators and mediators, OR (5) spatiotemporal aspects (Global positioning system, GPS, geographic information system, GIS, out of school hours). The entire search thesauruses and strings are available in online supplemental file 2. Additional papers were identified from the citation lists of individual and review papers.

\section{Eligibility criteria}

Studies were included for review if they were: published in peer-reviewed journals, in English, were observational studies that collected data directly on participants aged between five and 12 years (primary-school-aged children), assessed neighbourhood safety (personal and road safety) either as perceived by parents and/or children or measured (crime or road conditions), and reported or measured children's active mobility outside school hours. Included publications were not restricted to a single geographic location or a specific time. Studies were excluded for failing to meet the inclusion criteria. Systematic, scoping, or narrative reviews, opinion and editorial pieces, and other non-original research publications were also excluded.

\section{Study selection process}

Using search terms developed for this review, one reviewer (RZ) screened titles and abstracts to identify those works that met the inclusion criteria. Abstracts of the downloaded papers were then reviewed by two independent reviewers (RZ, CX) against the selection criteria. The final list of included studies was agreed by consensus involving a third reviewer (RN). Studies that met inclusion criteria were reviewed in full by RZ.

\section{Data extraction process}

The first author (RZ) extracted, into an excel datasheet relevant information on: author(s) year of publication, year of data collection, location of study, sample size (separated by gender), age/school grade, characteristics regarding methods of measuring or assessing neighbourhood safety (perceived or measured), children's active behaviour and outcome measures (eg, body mass index (BMI) or medium-to-vigorous physical activity (MVPA)), child's neighbourhood, other variables accounted in studies analysis including cofounders (eg, sex/gender/ family characteristics) and summary of findings. The data were collated into a systematic narrative summary table of the relevant papers. 
Patient and public involvement

No patients involved.

\section{Data synthesis}

Correlates between COAMB and safety were organised according to aspects of the socioecological framework (ie, individual, family, and neighbourhood). This framework is frequently used in active health behaviour research ${ }^{29}$ as a conceptualisation tool. This tool provides a structure for organising and understanding multiple factors that cause changes in health behaviour. Additionally, to enable the review investigating influences specific to forms for active behaviour, the outcome of correlates were compared by grouping findings into one of four categories: active travel; school travel; active play; active play, travel and exercise. The subgrouping corresponds to children's main types of structured and unstructured active behaviour outside of school hours. ${ }^{30}$

\section{Quality assessment}

Two independent reviewers (RZ, BJ) appraised the selected studies using priori defined quality criteria. Previously practised quality assessment by Schoeppe et $a l^{24}$ for active travel, Marzi and Reimers ${ }^{31}$ on independent mobility, and by Lubans $e t a l^{27}$ on active school travel, were reviewed. We adopted 13 existing criteria used earlier that covers all pertinent studies quality reporting characteristics. We expanded on six items to the checklist based on findings from a recent systematic review of which methodological measures in observational studies were assessed and found to be fundamental to increase reliability in children's active behaviour research. ${ }^{32}$ Those measures were covering: COAMB (objective or subjective), temporality elements such as change in time of day, weekdays or weekends (addressed in both perceptions of safety as well as COAMB), the delineation of the neighbourhood area (arbitrary or measured) and accounting for cofounders. We adopted the scoring system applied by Marzi and Reimers ${ }^{31}$ and Schoeppe $e t a l^{24}$ to quantify study robustness. Each criterion in the quality assessment checklist was given the assigned score if coded 'yes', 0 if coded 'no' or unclear, and half the score if coded 'partial'. A total overall score out of eight points was assigned, showing in table 1. As included studies have employed a variety of tools and sometimes composite techniques to measure active behaviour, we adopted Marzi et al method for criterion number fourteen that was scored on a scale ranging from 0 to 1 . For example, if a study employed an accelerometer and GPS, the study accumulates 0.5 points. However, when the study only used one measurement tool, it was assigned 0.25 points. A study variable that was measured appropriately but was not applicable to a specific criterion used for the quality assessment was discounted in the sum of total points used to derive quality score.$^{24}$ For example, the quality assessment criterion related to temporal and spatial measurement was not applicable for a paper where the primary outcome measure was BMI. In that instance, the criterion was removed from the total quality score of that paper. ${ }^{24}$ The quality score for each study was calculated by converting the total accumulated score into a percentage (total accumulated score $/ 8$ (maximum possible score) $\times 100) .{ }^{24}$ Adopting Schoeppe et al cut-off for levels of studies' quality, a percentage score of $\geq 66.7 \%$ was deemed robust, a score between $\geq 50$ and $<66.6 \%$ was considered moderate, and $<50 \%$ was rated poor.

\section{Evidence synthesis and strength}

Evidence synthesis described by Rothman et $a l^{22}$ and Ikeda $e t a l^{20}$ was used in this review. Each correlation and its direction (positive $(+)$, negative $(-)$, or inconclusive/ no correlation $(0))$ was identified and coded in terms of statistically significant or non-significant findings organised by the socioecological levels. To facilitate synthesis of evidence, we performed a reverse coding of the original direction of associations (ie, positive to negative or vice versa) as necessary, depending on the wording used in each work. ${ }^{20} \mathrm{~A}$ minimum of five studies with significant or non-significant findings was adopted to generate a consensus. ${ }^{20} 22$ Evidence with less than five studies available for synthesis was rated as limited, ${ }^{24}$ and with three studies was rated insufficient. Deriving strength of evidence was also adapted from previous reviews ${ }^{24} 27$ using the proportion and quality of studies reporting a significant association. The ratio of studies found with robust quality in this review to those found in an earlier review $^{24}$ was used to rescale the quality thresholds. In a minimum of five studies, if $\geq 27 \%$ of the total synthesised significant evidence that agrees in a direction (ie,,+- or 0 ) was of robust studies, the evidence was rated strong, from $\geq 20 \%-<27 \%$ was moderate strength, and at $<20 \%$ was rated weak.

\section{RESULTS}

\section{Study selection}

Following the title screening, a total of 231 papers across the six databases were initially identified that deemed potentially relevant. After removing 128 duplicates, the abstract review resulted in 64 articles that underwent a full-text review. Seven additional studies were identified from a manual search of individual reference lists. Following full-text review, a total of 29 studies met inclusion criteria, figure 1.

\section{Study characteristics}

The characteristics of the 29 studies, including settings, measurement methods and evidence output, are summarised in table 1 in online supplemental file 2. There were three longitudinal studies, ${ }^{1033} 34$ and the remaining ( 25 studies: $86 \%$ ) had a cross-sectional design. Twelve of the 29 studies (41\%) featured analysis using data from other larger scale or government projects (eg, the Children Living in Active Neighbourhoods study, ${ }^{35} 36$ the Built Environment and Active Transport project ${ }^{37}$ and the Spatio-Temporal Exposure and Activity Monitoring (STEAM) project $^{38}$ ). Populations varied across 
Table 1 Criteria for quality assessment and scoring for each criterion adapted from previous published systematic reviews

\section{Criteria}

Adopted quality assessment characteristics

1. Study objectives

2. Design

3. Target population

4. Random sampling

5. Study participant number

6. Participants' inclusion/exclusion

7. Study population

8. Participant recruitment

9. Response rate

10. Data collection

11. Data sources

12. Missing data

13. Statistical method

14. Active behaviour measures
15. Temporal active behaviour measures

16. perceived safety measures characteristics

17. Area of exposures

18. Evidence depicted temporal variation

19. Adjustments (Cofounders)

\section{Description}

Are objectives clear?

Was the study design appropriate for research undertaken?

has the target population defined?

Was a random sampling of the target population taken or was sampling appropriate for the study design?

Was the number of participants at each stage reported?

Were criteria for inclusion and exclusion of the participants used?

Was the study population sufficiently described, that is, sample size, gender, age, indicators of socioeconomic status?

Was participant recruitment described or referred to?

Was the response rate $60 \%$ or more?

Did the study describe data collection, that is, by mail, by interview, objective measure?

Did the study describe source of data, that is, questionnaire, survey, focus group, accelerometer, GPS?

Were numbers/percentages of the participants with missing active behaviour data reported, and did at least $80 \%$ of enrolled participants provide complete data to include?

Was it clear what was done to determine statistical or Yes $=0.5$ spatial statistical significance, for example, $\mathrm{p}$-value, $\mathrm{Cl}$ ? $\mathrm{No}=0$

Did the study objectively measure active mobility, that is, activity tracking. Spatial technology or web application?

Were measures reliable and valid for subjective measures?

Quality score per criterion

(Yes $=0.25$-point $\mathrm{No}=0$ of each criterion)

(Yes $=0.25$-point $\mathrm{No}=0$ of each criterion)

(Yes=0.25-point $\mathrm{No}=0$ of each criterion)

GPS $=0.25$

(accelerometer) $=0.25$ questionnaire $=0.25$

Travel diary $=0.25$

N/A

Was measured active behaviour related to temporal characteristics, that is, weekends vs weekdays or before and after school?

Yes $=0.5 / \mathrm{No}=0$

N/A

Did the study account for spatial or temporal features of Yes=0.5

the safety feelings? (Weekdays vs weekends or daytime No=0

(before and after school)) Or was measured safety conducted using geocoding?

Did the study delineate the exposed area

'neighbourhood' objectively or evaluate arbitrarily?

Objectively $=0.5$

Arbitrary $=0$

$\mathrm{N} / \mathrm{A}$

Yes $=1 / \mathrm{No}=0$

behaviour of evidence?

Did the study account for cofounders of age, sex, ethnicity, and family characteristics?
Each is $0.25 /$ Total $=1$ studies, with both parents and children represented. The sample sizes ranged from 35 children $^{6}$ to 31000 households. ${ }^{39}$ Studies alternatively used age groups or school grade to refer to children's age, but the mean ages were within the specified range of 5-12 years. Included works examined associations between forms of COAMB and perceived safety (21 of 29 studies; $73 \%$ ), measured safety (5 of $29 ; 17 \%$ ) or both measured and perceived safety

GPS, global positioning system. 


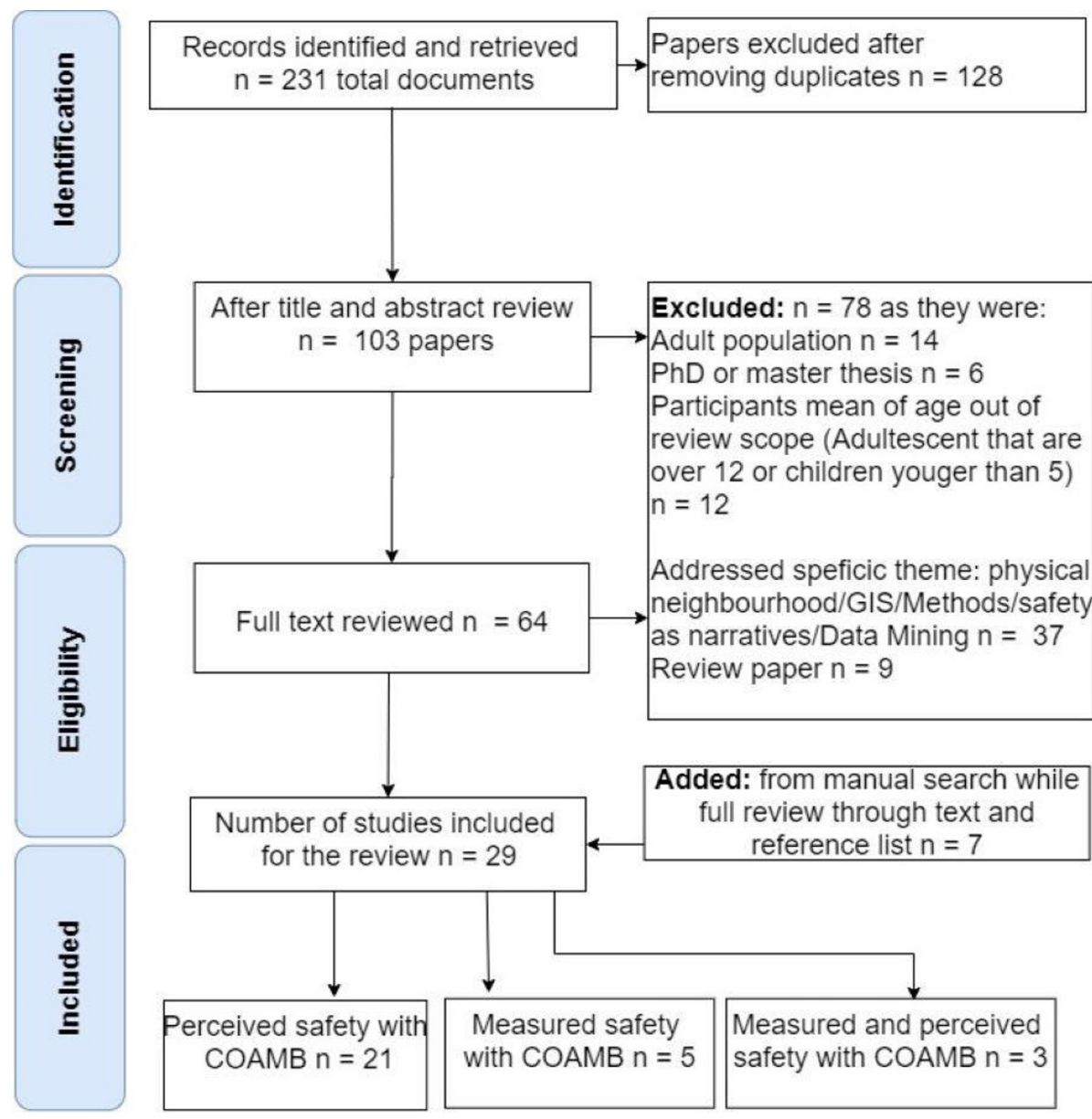

Figure 1 Preferred Reporting Items for Systematic Reviews and Meta-Analyses flow diagram for scoping review of neighbourhood safety correlates to children's outdoor active mobility behaviour (COAMB). GIS, geographic information system.

(3 of 29; 10\%). Collectively, the publications spanned Asia, Europe, North America and Oceania, Diagram 1 of online supplemental file 2. Aside from two Iranian studies, ${ }^{40}{ }^{41}$ and one from Malaysia, ${ }^{42}$ the remaining publications (26 of 29; 90\%) addressed populations in developed countries, with five each from the USA ${ }^{34} 43-46$ and the UK $\mathrm{UK}^{1047-49}$; four each from Canada ${ }^{533750}$ and Australia $^{35365152}$; two each from New Zealand ${ }^{5354}$ and the Netherlands, ${ }^{755}$ and one each from Portugal,${ }^{56}$ Finland $^{6}$ and Austria. ${ }^{57}$ Except for one study, ${ }^{58}$ remaining papers were published between 2007 and 2020. Two studies used $\mathrm{BMI}^{3448}$ as a measure of children's active health indices, yet one article has combined BMI with self-reported physical activity. Subjective assessment was the most common method of measurement of children's active behaviour. Ten of 29 studies (34\%) employed objective measures such as accelerometers, ${ }^{35} 3645$ pedometers,${ }^{52}$ GPS $^{6}$ alone or in combination with an accelerometer, ${ }^{37} 5053$ or GIS loggers. ${ }^{33}$ In view of the heterogeneity in the measurement methods, disparity in studies' units of outcome were apparent as exhibited in Diagram 2 of online supplemental file 2. Of 24 studies which examined personal safety, the majority assessed safety as perceived by parents; however, 7 studies featured safety perception among children. ${ }^{5414749515258}$ Five studies 710365053 examined objective measures of road safety and 12 studies assessed road danger as perceived primarily by parents. The findings in 25 of the 29 studies $(86 \%)$ were reported separately for boys and girls.

\section{Methodological quality assessments and strengths in evidence}

Nine of 29 studies (31\%) were rated of robust quality, 16 studies $(55 \%)$ were of moderate, and four $(14 \%)$ were rated as poor quality, as presented in table 2 in online supplemental file 2. Most studies focus on correlates of safety to active travel behaviour, such as independent walking or cycling to local destinations. Table 3 in online supplemental file 2 showed that active school travel was the focus in seven of 29 (24\%), ten of 29 (34\%) addressed active travel, and seven of 29 (24\%) addressed active play, active school travel and exercise. Active play alone was investigated in 3 of $29(10 \%)$ studies, and two (8\%) used BMI, but one study combined BMI with self-reported physical activity, figure 2 .

\section{Synthesis of the evidence}

Derived from table 4 in online supplemental file 2, we summarised below statistically significant and 


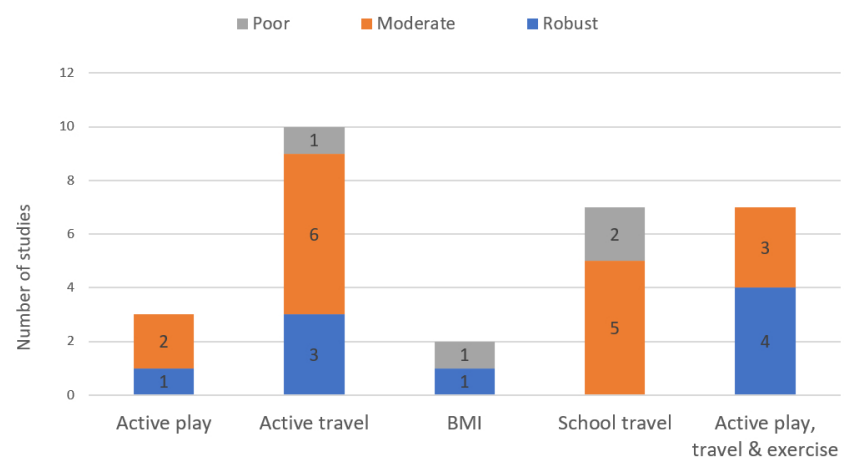

Figure 2 Studies grouped by the type of active behaviour indicating studies' level of methodological quality assessment. The $\mathrm{x}$-axis represents studies as grouped by type of active behaviour examined in each study. $y$-axis denotes the number of studies giving the different levels of evidence 'strength' when total methodological review score was summed. Strength of evidence was robust when summing total score was $\geq 66.66 \%$ moderate sum was $\geq 50$ $<66.6 \%$ and poor when an accumulated score was $<50 \%$. Adapted from previous review by Schoeppe et al. ${ }^{24}$

non-significant correlations in 29 studies examining influences on COAMB by aspects of socioecological levels that were.

\section{Individual level}

Without considering safety and supported by strong evidence $(40 \%$ in 10 out of 15 studies reporting significance and agrees in the direction were of robust quality), we found a correlation between sex/gender and COAMB. A higher level of COAMB was accumulated by males than female children, figure 3. Boys had more active behaviour, ${ }^{44}$ took more active trips, ${ }^{35}$ accessed larger activity spaces, ${ }^{52}$ and increased their active travel of walking or cycling to school, ${ }^{10}$ or other destinations. ${ }^{52}$ However, this correlates influenced by: temporal variation between weekdays and weekends, ${ }^{37}$ time segments of the day (after dark) ${ }^{51}{ }^{52}$ or actual distance travelled. ${ }^{6}$ Similarly, strong evidence $(33.3 \%$ in 6 studies reporting a significant positive correlation were of robust quality) showed that children of older age groups or higher school grades accumulated more COAMB.

\section{Family level}

Eight studies found that correlates of COAMB and car ownership were consistently associated with lower levels of COAMB. Despite that, the level of evidence was weak, with only $12.5 \%$ of the total studies were robust quality. The inconsistent finding across five studies suggests no clear correlation between ethnicity/race or socioeconomic status (SES) and COAMB. Children of minority groups in the UK achieved lower levels of active behaviour, ${ }^{47}$ whereas children of minority groups in the USA accumulated higher levels of active behaviour. ${ }^{44}$ In New Zealand, children's active behaviour differed by race/ethnicity and the day of the week: Indian/Asian background had increased their physical activity levels of medium-tovigorous (MVPA\%) on weekends while Pacific, European

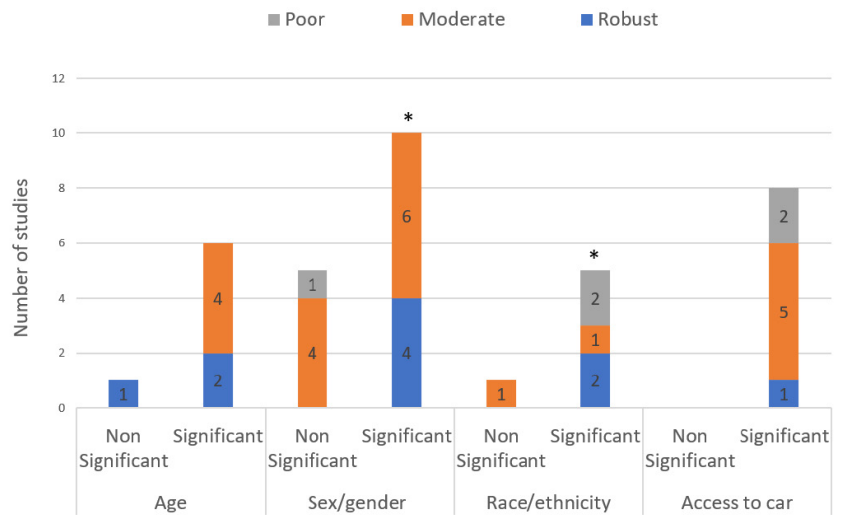

Figure 3 Correlation between individual (child) and family characteristics and children's active mobility behaviour. The $\mathrm{x}$-axis represents variables that accumulated the minimum number of studies to synthesise evidence of correlates between COAMB and sex/gender, age, access to a car, and ethnicity. $y$-axis denotes the number of studies that examined the correlation showing accumulated significance or nonsignificance. Note: *=in sex/gender, we saw variability by temporal characteristics (ie, changes between weekdays and weekends or time of the day (before and after school)) in three studies and reduced COAMB by distance travelled in one study. ${ }^{*}=$ In Race/ethnicity, one study with a significant correlate depicted temporal change (i.e., weekend/ weekdays).

and Maori children accumulated higher (MVPA\%) on weekdays. ${ }^{53}$ Findings on (SES) and COAMB were either: non-significant for female children (5-6years); increased active behaviour in high (SES) groups ${ }^{4251}$; inconclusive ${ }^{59}$; and increased BMI level in a high deprivation area yet reported higher self-physical activity. ${ }^{48}$

\section{Neighbourhood level}

We found strong evidence that increased parental perception of safety correlated with lower COAMB (29.4\% of the 17 out of 18 studies reporting significant and agreeing in the direction were of robust quality), figure 4 . We found limited and inconsistent evidence on the correlation of 'stranger danger' among parents to COAMB; no relationship, ${ }^{546}$ reduced MVPA, ${ }^{45}$ and has a temporal correlation (son weekdays). ${ }^{37}$ We found moderate evidence $(25 \%$ of the 11 studies were rated robust) but consistent in the direction of correlates of higher parental road safety concerns and decreasing levels of COAMB, including one study finding a gender difference (girls). Weak evidence yet consistent (i.e., agree in the direction of the significance) of lower levels of COAMB correlate with children's increased perception of danger from strangers, roads and personal safety. Limited (four studies) evidence related to children's perception of personal safety, ${ }^{5} 414952$ included a study that depicted variation by gender. ${ }^{52}$ Perception of 'stranger danger ${ }^{515560}$ was insufficiently (three studies) addressed. Road danger ${ }^{474515258}$ depicted variation in two studies by sex/gender. ${ }^{49} 58$ Regarding measured safety (i.e., crime 


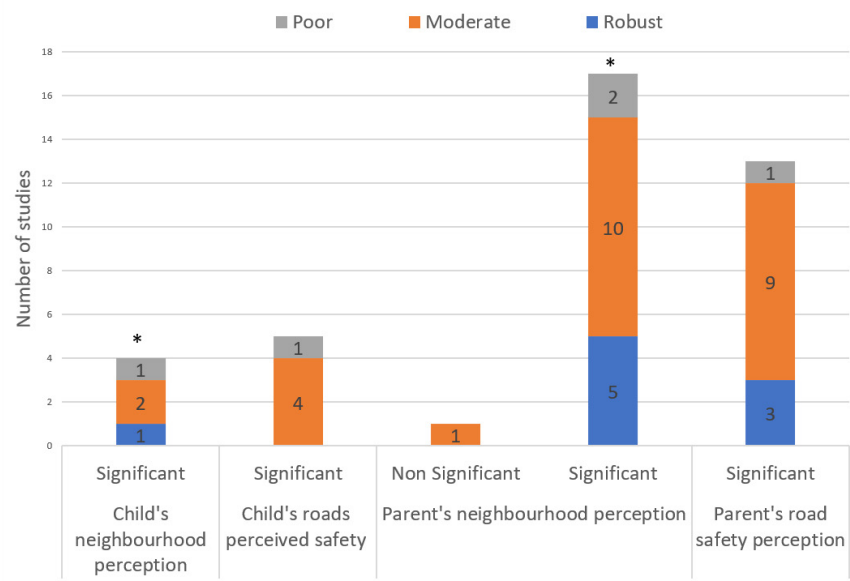

Figure 4 Perceived safety (parents and children) of personal and road danger correlates to reported and measured children's active mobility behaviour. The x-axis represents examined variables that accumulated a minimum number of studies to synthesise evidence for perceived personal and road safety by parents or children. $y$-axis denotes the number of studies that accumulated significant or non-significance. Note:*=Parents' perceived neighbourhood safety showing significant correlates included three studies depicting temporal characteristics (weekdays/weekends or before and after school), three studies varied by child's sex/gender and one study by age. *=For children, perceived personal safety varied by gender in one study.

or road conditions) and COAMB, a definitive conclusion cannot be drawn despite robust evidence (six out of nine studies are of robust quality),. A synthesis of four studies showed inconsistency in the direction of the relationship with measured personal safety, ${ }^{33} 344546$ as one study depicted increased active transportation in high crime areas. ${ }^{33}$ Road danger ${ }^{3} 71050$ showed increased outdoor active play in highest traffic areas, ${ }^{50}$ and one study lacked correlation to measured roads environment. ${ }^{36}$ Other characteristics in the neighbourhood that are likely to be influenced by perceived safety were: strong evidence of distances travelled (perceived or objectively measured) with 10 studies and $40 \%$ of robust quality and variability in neighbourhood type (6 studies with $66.6 \%$ of robust quality). Other addressed variables demonstrated associations with COAMB, but insufficient evidence to conclude are in table 4 of online supplemental file 2.

\section{DISCUSSION}

A review of 29 studies investigating multifaceted influences on COAMB and neighbourhood safety prompted the discussion over three primary topics.

\section{Inequality in opportunity for children's active behaviour}

Differences in individual, family, and neighbourhood safety, created an unequal opportunity for COAMB that was ubiquitous across geographical regions, Diagram 3 of online supplemental file 2. Our review depicted variability of COAMB by sex/gender that was predominant in active play and exercise forms of active behaviour than in active travel. This explains lacking of agreement with previous reviews on independent mobility ${ }^{61}$ and active school travel, ${ }^{22}$ yet agreed with reviews on physical activity. ${ }^{1562}$

Our review suggest that with more access to cars, parents conveniences, ${ }^{22}$ or other influences such as weather conditions, children at young age are more likely, regardless of their sex/gender, to be driven more often than actively travelling (eg, school trips). However, variability in sex/gender becomes more apparent in physical activity as it is an elective form of active behaviour behind school or government jurisdiction. Increased COAMB with age undetected in previous reviews ${ }^{152}$ could be owned to earlier reviews combining a broad range of children's age (3-12 years ${ }^{61}$ or 3-18 years ${ }^{15}$ ). A longitudinal study suggested that some forms of active travel (such as school trip) become more prevalent with age, starting from 6 years. ${ }^{60}$

Studies variability restrained clear conclusions on family race/ethnicity or neighbourhood (SES) correlates to COAMB also concord with earlier reviews findings. ${ }^{20} 2$ Children from low SES areas in the $\mathrm{UK}^{48}$ were more active. However, children from wealthy families in Australia and Malaysia $^{42}{ }^{51}$ had increased active behaviour. Children from less affluent areas may be active by necessity, whereas more accessibility opportunities to open spaces and recreational centres are offered to children from high (SES) areas. A study on obesity rates showed that migrant children (in lower SES) were more active than resident children with higher (SES). ${ }^{63}$ This socioeconomic influence appeared to reverse its effect on COAMB by geographical location (between developed and developing countries). Nevertheless, we found variation in the correlates of COAMB to (SES) by gender (female children), ${ }^{515560}$ age group $^{55}$ and higher income people feeling safer, which in turn encouraged active school travel. ${ }^{55}$ Despite an agreement with earlier reviews, ${ }^{20} 22$ the decline in COAMB concerning family ownership of car conflicts with some studies suggesting that wealthier families are more active, thus calling for further investigation.

Every child is entitled to feel safe at all times. Nevertheless, findings of this review suggest a distinct inequality as children living in low perceived safe areas are declined the opportunity to be active. Our finding conflicts with two previous reviews on active travel ${ }^{16}$ and physical activity, ${ }^{15}$ respectively. The broad range of children age addressed in the two reviews may have contributed to the disagreements in findings. Parents have a larger influence on younger children's active health behaviour ${ }^{9}$ and perhaps hold greater fears regarding road safety for younger children that may not contribute significantly in the older age group, as was explained by a longitudinal national study in the US. ${ }^{64}$ However, concerns regarding personal safety are nuanced by gender (females), ${ }^{6} 3552$ temporal variations (on weekdays only ${ }^{53}$ or weekends, ${ }^{35}$ and intersect with gender (females) and temporality when after dark. ${ }^{6}$ The prevalence effect of 'stranger danger' on COAMB among studies surveying children suggests that vulnerability to strangers' harm is more significant for children, particularly for girls. 


\section{Influences on COAM are specific to the type of active behaviour}

Child's sex/gender is correlated to COAMB, but most apparent in active play and physical activity rather than active travel. This review also depicted that perceived personal safety risk is most significant in restricting children's active travel, limiting their active space. This finding concurs with earlier research revealing shrinking children's active space with the rise of personal safety fears. ${ }^{2}$ Nevertheless, parents' concerns over road danger restricted all types of COAMB, Diagram 4 of online supplemental file 2. More active travel was regarded in the urban residential and commercial neighbourhood, was also concluded previously ${ }^{31}$ suggesting that dense urban area may offer a sense of safety, encouraging more active travel among children. The correlate was also confirmed by the reversed influence of reduced active travel correlates to perceived or measured distance occurred with changes to roads and infrastructure..$^{55}$

\section{Methodological challenges}

The inconsistencies seen in studies investigating neighbourhood safety correlates to COAMB, limit the ability to draw definite conclusions in some areas. Our methodological quality assessment revealed that most studies fulfilled elements relevant to studies reporting but essentially lacked addressing components in the design, method of measurement, and analysis for evidence-based research on children's active health. ${ }^{32}$ This may have resulted in the majority of studies were of moderate and poor quality. Subjective measures using questionnaires rely on human recall distant events. ${ }^{65}$ Yet, we found some inconsistencies among studies employing objective measures. Although a review of measurement methods is out of the scope of this paper, the discrepancies in some of the outcome necessitate highlighting some critical research challenges for future research attention yet were elaborated further elsewhere by Zougheibe $e t a l{ }^{32}$ Study design is likely to affect the outcome. Longitudinal studies limit selection bias associated with cross-sectional design. Inconsistency in the direction of correlates among studies employed objective measures showed most problematic measurement methods were in (1) inconsistent inclusion of crime categories for measured personal safety studies or elements addressed roads conditions, (2) measuring COAMB using spatial activity tracking (i.e., GPS) was faced with an absence of standardised measurement protocols that caused variabilities in few areas such as the inclusion of surveyed days and threshold of counts per minutes of registered data, that could impact a true interpretation of results, (3) the spatial extent of the child's neighbourhood to derive safety exposures was primarily defined arbitrarily. Still, studies that adopted spatial measures had employed diverse methods, (4) inconsistent accounting for cofounders and (5) infrequent accounting for temporal safety and behaviour characteristics. We found scarcity in research on correlates of COAMB to variation of safety perceived by parents to children residing in the same neighbourhood and actual crime.

\section{Implications of findings}

Successful implementation of any intervention strategy to encourage parents and children to embrace an active mobility culture require collaboration among all levels of children, families, schools, community-based organisations, city planners, policy-makers and researchers. ${ }^{28}{ }^{66}$ The revealed multiple influences contributing to children's active behaviour guided our recommendations below.

\section{Interventions for vulnerable groups of the population}

Ignoring gendered primary-school-aged children's active mobility behaviour may result in only male adult's regarding physical activity as essential or accessible. Therefore, strategies to increase active behaviour must be sex/gender and age specific. Additionally, the indication of influence on COAMB by diverse (SES) and race/ethnic backgrounds suggests that a subgroup of the population is constantly inactive. Parental involvement in educational programmes to promote children's free play and active travel or children's encouragement in school and community programmes to participate in active travel and leisure active play could be useful. Earlier evidence also supports the positive influences of active parents ${ }^{56}$ or positive peer influence. ${ }^{67}$

\section{Promote child-friendly cities}

Revitalise cities design may offer play opportunity and safer travel for children. Perceived low safety linked to declined children's active travel and its associate to neighbourhood types suggests that current cities and roads designs are maybe seen as unsafe for children to be outdoors actively playing and travelling safely to local destinations independently. Most importantly, interventions intended to encourage children to travel further actively call for redesign of urban structures to enable active travel and safety at the same time. This may include upgrading local features that promote walkability in desirable neighbourhood characteristics (eg, schools, shops, libraries) and active playing in local parks or exercise in an 'activity-friendly neighbourhood'. ${ }^{68}$ More voices are calling for children and families to be included in designing new urban spaces. Improvements in road safety may increase overall COAMB and enable children and families to embrace an active mobility culture. Existing streets design is seen as unsafe and unappealing for children and their parents. Prompt policy interventions to address most concerned roads conditions(eg, heavy traffic, fast drivers, high-speed roads, lack of road signals, availability of sidewalks and cycling infrastructure) may alleviate parents' and children's concerns over road danger.

\section{Advancing research}

Methodological improvements to reduce inconsistency in the outcome and increase reliability in future research can better inform evidence-based policy. Namely: encouraging longitudinal study design and adopting a three-dimensional conceptual framework in research as suggested earlier ${ }^{32}$ to account for critical elements of (1) what we measure: the determinants that affect the outcome and consistent accounting 
for cofounders (sex/gender, SES background, and ethnicity/race background), (2) how we measure: involves frequency and intensity for perceived safety ${ }^{69-71}$ (personal and roads) and active behaviour whether reported or measured consistency in addressing crimes only relevant to personal safety, and (3) spatiotemporal characteristics (i.e., locality of active behaviour or safety incident, spatial extents of children neighbourhoods, and time) ${ }^{72}$ Further research on COAMB correlates to variability in parents to their children safety perception or measured to perceived safety, and inequality in the neighbourhood (SES) would answer more profound research questions.

\section{STRENGTHS AND LIMITATIONS}

This review was limited to English-language and peerreviewed observational studies. Despite every effort to identify all relevant studies through a variety of terms used, some relevant studies may have been omitted due to multiple synonyms used in works. The observed heterogeneity in study measures prevented a straightforward meta-analysis; thus, we conducted quantitative vote counting to overcome existing disparities in outcome and methods. The majority of studies were cross-sectional design and were of moderate or poor quality. Despite these limitations, this review has important strengths. This work synthesised findings and derived a quantitative understanding of evidence strength by combining a measurement of study reporting quality, involving critical characteristics that may improve reliability in future observation studies. This paper has comprehensively addressed the impact of safety in primary-school-aged children's active behaviour, eliminating inconsistencies resulting from a broad age range of study participants. To the best of our knowledge, this review is the first to consider the impact of perceived (by parents and children) and measured neighbourhood safety across multiple types of COAMB. Finally, this review was not limited to publication date or geographical region.

\section{CONCLUSION}

This systematic review of evidence revealed that there is inequality in COAMB by children's sex/gender and age and some indication regarding race/ethnicity and (SES) despite the evidence being inconclusive with regard to the latter two examined variables. Compared with safer neighbourhoods, children living in perceived unsafe areas correlated with lower outdoor active mobility behaviour and reduced active play. However, perceived personal safety risk has primarily restricted children's active travel to local destinations, whereas perceived danger from traffic reduced every type of COAMB. Nevertheless, the direction and strength of the correlates are affected by individual and family characteristics, distance travelled, and time (weekend/weekday/time of day). These findings were consistent across countries. There is a need to use validated measurement methods. Deeper understanding of safety (perceived or measured) correlates to race/ethnicity, (SES) variances and COAMB (spatial extent of active behaviour or intensity of physical activity) may answer more profound behavioural research questions. Current inequalities in children's opportunities to engage in active mobility behaviour require sex/gender and age-based interventions. Most importantly, interventions aimed at improving personal safety and engaging children in urban design to promote child-friendly cities may prompt children to travel further actively. Improvements in road conditions may increase overall COAMB and enable children and families to embrace an active mobility culture.

Acknowledgements The authors thank Jianhong (Cecilia) Xia (CX) for her assistance in assessing studies for inclusion. Additionally, the authors would like to thank the thoughtful comments made by the reviewers and editor from BMJ Open Journal that have helped improve the review considerably.

Contributors RZ carried concept/design, data retrieval, screening of the literature for inclusion, quality rating, synthesised the findings, drafted, and finalised the manuscript. BJ assisted in rating of studies quality and contributed to proof editing the overall manuscript. $\mathrm{RN}, \mathrm{OG}$ and $\mathrm{AD}$ reviewed and approved the manuscript.

Funding The first author receives the Australian Government Research Training Program (RTP) scholarship; however, the research received no specific grant from any funding agency in public, commercial or not-for-profit sectors.

Competing interests None declared.

Patient consent for publication Not required.

Provenance and peer review Not commissioned; externally peer reviewed.

Data availability statement All data relevant to the study are included in the article or uploaded as supplemental information.

Supplemental material This content has been supplied by the author(s). It has not been vetted by BMJ Publishing Group Limited (BMJ) and may not have been peer-reviewed. Any opinions or recommendations discussed are solely those of the author(s) and are not endorsed by BMJ. BMJ disclaims all liability and responsibility arising from any reliance placed on the content. Where the content includes any translated material, BMJ does not warrant the accuracy and reliability of the translations (including but not limited to local regulations, clinical guidelines, terminology, drug names and drug dosages), and is not responsible for any error and/or omissions arising from translation and adaptation or otherwise.

Open access This is an open access article distributed in accordance with the Creative Commons Attribution Non Commercial (CC BY-NC 4.0) license, which permits others to distribute, remix, adapt, build upon this work non-commercially, and license their derivative works on different terms, provided the original work is properly cited, appropriate credit is given, any changes made indicated, and the use is non-commercial. See: http://creativecommons.org/licenses/by-nc/4.0/.

\section{ORCID iD}

Roula Zougheibe http://orcid.org/0000-0002-3649-583X

\section{REFERENCES}

1 Steinbeck KS. The importance of physical activity in the prevention of overweight and obesity in childhood: a review and an opinion. Obes Rev 2001;2:117-30.

2 Jack G. Place matters: the significance of place attachments for children's well-being. Br J Soc Work 2010;40:755-71.

3 Carver A, Timperio A, Crawford D. Playing it safe: the influence of neighbourhood safety on children's physical activity. A review. Health Place 2008;14:217-27.

4 Stone MR, Faulkner GE, Mitra R, et al. Physical activity patterns of children in Toronto: the relative role of neighbourhood type and socio-economic status. Can J Public Health 2012;103:S9-14.

5 Loebach JE, Gilliland JA. Free Range Kids? Using GPS-derived activity spaces to examine children's neighborhood activity and mobility. Environ Behav 2016;48:421-53. 
6 Fagerholm N, Broberg A. Mapping and characterising children's daily mobility in urban residential areas in Turku, Finland. Fennia 2011;189:31-46.

7 Helbich M, Emmichoven MJZvan, Dijst MJ, et al. Natural and built environmental exposures on children's active school travel: a Dutch global positioning system-based cross-sectional study. Health Place 2016;39:101-9.

8 Lia K, Willem van V x. Children in the city: Reclaiming the street. Children, Youth and Environments 2006;16:151-67.

9 McMillan TE. Urban Form and a Child's Trip to School: The Current Literature and a Framework for Future Research. J Plan Lit 2005;19:440-56.

10 Carver A, Panter JR, Jones AP, et al. Independent mobility on the journey to school: a joint cross-sectional and prospective exploration of social and physical environmental influences. J Transp Health 2014;1:25-32.

11 Donovan J. Designing the compassionate City to overcome built-in biases and help us live better La Trobe university: Routledge. 2018 [ $2020 \mathrm{https}$ ///theconversation.com/designing-the-compassionatecity-to-overcome-built-in-biases-and-help-us-live-better-92726

12 Schwab K. Play access in cities is critical to children's 2020.

13 Garofalo J. The fear of crime: causes and consequences. J Crim Law Criminol 1981;72:839.

14 Lorenc T, Petticrew M, Whitehead M, et al. Crime, fear of crime and mental health: synthesis of theory and systematic reviews of interventions and qualitative evidence. Public Health Res 2014;2:1-398.

15 Davison KK, Lawson CT. Do attributes in the physical environment influence children's physical activity? A review of the literature. Int $J$ Behav Nutr Phys Act 2006;3:19.

16 Panter JR, Jones AP, van Sluijs EM. Environmental determinants of active travel in youth: a review and framework for future research. Int $J$ Behav Nutr Phys Act 2008;5:34.

17 WHO. Global status report on non communicable diseases 2010 2010;176.

18 Pellis SM, Pellis VC, Bell HC. The function of play in the development of the social brain. Am J Play 2010;v2:p278-96.

19 Council loMNR. From neurons to neighborhoods: the science of early childhood development. Washington, DC: The National Academies Press, 2000.

20 Ikeda E, Hinckson E, Witten K, et al. Associations of children's active school travel with perceptions of the physical environment and characteristics of the social environment: a systematic review. Health Place 2018:54:118-31.

21 Wong BY-M, Faulkner G, Buliung R. GIS measured environmental correlates of active school transport: a systematic review of 14 studies. Int J Behav Nutr Phys Act 2011;8:39.

22 Rothman L, Macpherson AK, Ross T, et al. The decline in active school transportation (AST): a systematic review of the factors related to AST and changes in school transport over time in North America. Prev Med 2018;111:314-22.

23 Pang B, Kubacki K, Rundle-Thiele S. Promoting active travel to school: a systematic review (2010-2016). BMC Public Health 2017; 17:638

24 Schoeppe S, Duncan MJ, Badland H, et al. Associations of children's independent mobility and active travel with physical activity, sedentary behaviour and weight status: a systematic review. J Sci Med Sport 2013;16:312-9

25 Pont K, Ziviani J, Wadley D, et al. Environmental correlates of children's active transportation: a systematic literature review. Health Place 2009;15:849-62.

26 Davison KK, Werder JL, Lawson CT. Children's active commuting to school: current knowledge and future directions. Prev Chronic Dis 2008;5:A100.

27 Lubans DR, Boreham CA, Kelly P, et al. The relationship between active travel to school and health-related fitness in children and adolescents: a systematic review. Int J Behav Nutr Phys Act 2011;8:5 http://dx.doi.org.dbgw.lis.curtin.edu.au/

28 Australian Bureau of Statistics. Australian health survey: physical activity, 2011-12. Canberra, 2013.

29 Mehtälä MAK, Sääkslahti AK, Inkinen ME, et al. A socio-ecological approach to physical activity interventions in childcare: a systematic review. Int J Behav Nutr Phys Act 2014;11:22.

30 Borghese MM, Janssen I. Development of a measurement approach to assess time children participate in organized sport, active travel, outdoor active play, and curriculum-based physical activity. BMC Public Health 2018;18:396.

31 Marzi I, Reimers A. Children's Independent Mobility: Current Knowledge, Future Directions. and Public Health Implications 2018;2018:2441.
32 Zougheibe R, Xia JC, Dewan A, et al. Children's outdoor active mobility behaviour and neighbourhood safety: a systematic review in measurement methods and future research directions. Int $J$ Health Geogr 2021;20:2

33 Vonderwalde M, Cox J, Williams GC, et al. Objectively measured crime and active transportation among 10-13year olds. Preventive Medicine Reports 2019;2019:48-51.

34 Suminski RR, Robson SM, May LL, et al. Actual neighborhood-level crime predicts body mass index z-score changes in a multi-racial/ ethnic sample of children. Prev Med Rep 2018;12:164-9.

35 Carver A, Timperio A, Hesketh K, et al. Are children and adolescents less active if parents restrict their physical activity and active transport due to perceived risk? Soc Sci Med 2010;70:1799-805.

36 Carver A, Timperio AF, Crawford DA. Neighborhood road environments and physical activity among youth: the clan study. $J$ Urban Health 2008;85:532-44.

37 Faulkner G, Mitra R, Buliung R, et al. Children's outdoor playtime, physical activity, and parental perceptions of the neighbourhood environment. International Journal of Play 2015;4:84-97.

38 Loebach J, Gilliland J. Neighbourhood play on the endangered list: examining patterns in children's local activity and mobility using GPS monitoring and qualitative GIS. Child Geogr 2016;14:573-89.

39 Waygood EOD, Susilo YO. Walking to school in Scotland: do perceptions of neighbourhood quality matter? IATSS Research 2015;38:125-9.

40 Mehdizadeh M, Mamdoohi A, Nordfjaern T. Walking time to school, children's active school travel and their related factors. Journal of Transport \& Health 2017;6:313-26.

41 Shokoohi R, Hanif NR, Dali MM. Children walking to and from schoo in tehran: associations with neighbourhood safety, parental concerns and children's perceptions. Procedia Soc Behav Sci 2012;38:315-23.

42 Tung SEH, Ng XH, Chin YS, et al. Associations between parents' perception of neighbourhood environments and safety with physical activity of primary school children in Klang, Selangor, Malaysia. Child Care Health Dev 2016;42:478-85.

43 Oluyomi AO, Lee C, Nehme E, et al. Parental safety concerns and active school commute: correlates across multiple domains in the home-to-school journey. Int J Behav Nutr Phys Act 2014;11:32.

44 Roberts JD, Knight B, Ray R, et al. Parental perceived built environment measures and active play in Washington DC metropolitan children. Prev Med Rep 2016;3:373-8.

45 Kneeshaw-Price SH, Saelens BE, Sallis JF, et al. Neighborhood Crime-Related safety and its relation to children's physical activity. $J$ Urban Health 2015;92:472-89.

46 Zhu X, Lee C. Walkability and safety around elementary schools economic and ethnic disparities. Am J Prev Med 2008;34:282-90.

47 Alton D, Adab P, Roberts L, et al. Relationship between walking levels and perceptions of the local neighbourhood environment. Arch Dis Child 2007;92:29-33.

48 Noonan RJ, Boddy LM, Knowles ZR, et al. Cross-Sectional associations between high-deprivation home and neighbourhood environments, and health-related variables among Liverpool children. BMJ Open 2016;6:e008693.

49 Page AS, Cooper AR, Griew P, et al. Independent mobility, perceptions of the built environment and children's participation in play, active travel and structured exercise and sport: the PEACH Project. Int J Behav Nutr Phys Act 2010;7:17.

50 Nguyen A, Borghese MM, Janssen I. Pedestrian traffic safety and outdoor active play among 10-13 year olds living in a mid-sized city. Prev Med Rep 2018;10:304-9.

51 Timperio A, Crawford D, Telford A, et al. Perceptions about the local neighborhood and walking and cycling among children. Prev Med 2004;38:39-47

52 Villanueva K, Giles-Corti B, Bulsara M, et al. How far do children travel from their homes? exploring children's activity spaces in their neighborhood. Health Place 2012;18:263-73.

53 Oliver M, Mavoa S, Badland $\mathrm{H}$, et al. Associations between the neighbourhood built environment and out of school physical activity and active travel: an examination from the kids in the City study. Health Place 2015;36:57-64

54 Lin E-Y, Witten K, Oliver M. Social and built-environment factors related to children's independent mobility: The importance of neighbourhood cohesion and connectedness. Health \& place $2017 ; 107$

55 van den Berg P, Waygood EOD, van de Craats I, et al. Factors affecting parental safety perception, satisfaction with school travel and mood in primary school children in the Netherlands. J Transp Health 2020;16:100837.

56 Santos MP, Pizarro AN, Mota J, et al. Parental physical activity, safety perceptions and children's independent mobility. BMC Public Health 2013;13:584-84. 
57 Stark J, Frühwirth J, Aschauer F. Exploring independent and active mobility in primary school children in Vienna. J Transp Geogr 2018;68:31-41.

58 Davis A, Jones L. Environmental constraints on health: listening to children's views. Health Educ J 1996;55:363-74.

59 Oliver M, McPhee J, Carroll P, et al. Neighbourhoods for active kids: study protocol for a cross-sectional examination of neighbourhood features and children's physical activity, active travel, independent mobility and body size. BMJ Open 2016;6:e013377.

60 Pabayo R, Gauvin L, Barnett TA. Longitudinal changes in active transportation to school in Canadian youth aged 6 through 16 years Pediatrics 2011;128:e404-13.

61 Ding D, Sallis JF, Kerr J, et al. Neighborhood environment and physical activity among youth a review. Am J Prev Med 2011:41:442-55.

62 Sallis JF, Prochaska JJ, Taylor WC. A review of correlates of physical activity of children and adolescents. Med Sci Sports Exerc 2000;32:963-75.

63 Liu W, Liu W, Lin R, et al. Socioeconomic determinants of childhood obesity among primary school children in Guangzhou, China. BMC Public Health 2016;16:482.

64 Datar A, Nicosia N, Shier V. Parent perceptions of neighborhood safety and children's physical activity, sedentary behavior, and obesity: evidence from a national longitudinal study. Am J Epidemiol 2013;177:1065-73.
65 Kelly P, Krenn P, Titze S, et al. Quantifying the difference between self-reported and global positioning Systems-Measured journey durations: a systematic review. Transp Rev 2013;33:443-59.

66 World Health organization. Equity, social determinants and public health programmes. 303, 2010.

67 Salvy S-J, Roemmich JN, Bowker JC, et al. Effect of Peers and friends on youth physical activity and motivation to be physically active. J Pediatr Psychol 2009;34:217-25.

68 Lee C, Moudon AV. Physical activity and environment research in the health field: implications for urban and transportation planning practice and research. J Plan Lit 2004;19:147-81.

69 Chataway ML, Hart TC. (Re)Assessing contemporary "fear of crime" measures within an Australian context. J Environ Psychol 2016;47:195-203.

70 Lorenc T, Petticrew M, Whitehead M, et al. Fear of crime and the environment: systematic review of UK qualitative evidence. BMC Public Health 2013;13:496.

71 Solymosi R, Bowers K, Fujiyama T. Mapping fear of crime as a context-dependent everyday experience that varies in space and time. Legal Criminol Psychol 2015;20:193-211.

72 Boessen A, Hipp JR, Butts CT, et al. Social fabric and fear of crime: considering spatial location and time of day. Soc Networks 2017;51:60-72. 